\title{
A Web Based Tool to Support a Personalized Therapeutic Path Through the Use of Psychological Tests
}

\author{
Elena LAZAROVA ${ }^{\mathrm{a}, ~}{ }^{1}$, Sara MORA ${ }^{\mathrm{a}}$, Davide ARMANINO ${ }^{\mathrm{b}}$, Alessandro POIRE ${ }^{\mathrm{b}}$, \\ Fabio FURLANI ${ }^{\mathrm{c}}$, and Mauro GIACOMINI ${ }^{\mathrm{a}}$ \\ a Department of Informatics, Bioengineering, Robotics and System Engineering \\ (DIBRIS), University of Genoa, Italy \\ ${ }^{\mathrm{b}}$ Genoa Constructivist Association, Genoa, Italy \\ ${ }^{\mathrm{c}}$ Como Cognitive Therapy Center, Via Rusconi 10, Como, Italy
}

\begin{abstract}
Psychology tests are tools used to evaluate specific aspects of individual psyche and behavior, in clinical practice and for research purposes. They are validated and standardized both in the administration procedures and interpretation of results. Nowadays, most of these tests are questionnaires administered to patients on paper support. The patient's answers to the questionnaires constitute a basis for self-presentation and self-awareness at the beginning of the therapeutic path. The computer is a valuable aid allowing a quickly consultation of all the answers and highlighting the most salient ones. The main aim of this work was to design, develop and test a computerized support tool for the interpretation of psychological tests that allow good interaction between groups of therapists sharing the same operating modes. The developed system allows: first the storing of the numerical values corresponding to the answers to the questionnaires of the patients; then it creates a complete 'Picture' of the patient and allows the automatic computation of the correlation between the indexes of the various scales. The graphical correlations between scales can be also a valuable aid in finding the outliers, so patients far away from the trend line.
\end{abstract}

Keywords. Psychological test interpretation, web based professional collaboration, correlation matrix, outliers identification, Z-score normalization

\section{Introduction}

Psychology tests are tools used to evaluate specific aspects of individual psyche and behavior, in clinical practice and for research purposes. They are validated and standardized both in the administration procedures and interpretation of results. They rely on statistical methods that allow the performance comparison between the tested group of people and the controls. Psychological tests can be divided into two main categories: personality tests, aimed at identifying the individual's personality traits, and efficiency tests, aimed at investigating the individual's knowledge and skills. Nowadays, most of these tests are questionnaires administered to patients on paper support. The patient can choose within a set of fixed answers. This choice, widely shared by virtually

${ }^{1}$ Corresponding Author: Elena Lazarova, Department of Informatics, Bioengineering, Robotics and System Engineering (DIBRIS), University of Genoa, Italy; E-mail: elena.lazarova@dibris.unige.it 
the entire scientific community of psychologists, is intended to obtain the most truthful possible answer with- out being influenced by the eventual subjection to any technological tool used to replace paper. The patient's answers to the questionnaires constitute a basis for self-presentation and self-awareness at the beginning of the therapeutic path. As the process goes on, the patient and the therapist review the most significant answers together, interpreting their meaning in an even deeper way, with knowledge and self-awareness that is broader and more meaningful for the patient himself. The computer, in its simplicity of operation and immediate usability, is a valuable aid allowing a rapid consultation of all the answers and highlighting the most salient ones. In this way the patient re-appropriates the symptom and his initial emotive discomfort by translating and progressively declining it as an emotional, cognitive and behavioral style of functioning. From the strangeness of the symptom, the patient moves on to understanding his personal initial functioning and grasps its adaptive, albeit painful and not optimal, meaning. The diagnostic tool, when strategically shared, becomes the basis of the therapeutic alliance based on transparency, sharing and mutual trust in the reading and understanding of the patient's way of being, as well as the direct object of therapeutic work along the way. This modality of setting up the therapeutic work also appears to be fully consistent with a dimensional vision of the diagnosis, overcoming an exclusively categorical, descriptive and nosographic approach. It is not a question of understanding the presence of a psychopathological syndrome but of grasping the patient's level of functioning (mobile, variable and slowly modifiable) along dimensions that describe styles and methods of adaptation. The main aim of the work presented here was to design, develop and test a computerized support tool for the interpretation of psychological tests that allow good interaction between groups of therapists sharing the same operating modes. The produced system allows the entry and orderly filling of clinical questionnaires for the evaluation of personality traits and dimensions, early maladaptive patterns, salient emotional and cognitive styles, as well as the main symptoms of the nosographic spectrum and the quality of the functioning of consciousness with respect to the presence, type, and quantity of dissociative symptoms in the patient. The set of these data composes a multifaceted and in-depth clinical "picture" of the patient immediately usable by the clinician, who consults it not only in the diagnostic phase while setting the therapeutic strategy but also along the treatment path, even directly during the psychotherapeutic session.

\section{Methods and Materials}

\subsection{Selected Psychological Tests}

As part of this research project, the included psychological tests are:

1. Young Schema Questionnaire version L3 (YSQ-L3): identifies maladaptive patterns and builds dynamic profile of the patient.

2. Personality Inventory Domain (PID-5): a personality inventory which supports diagnosis process according to the: Diagnostic and Statistical Manual of Mental Disorders (DSM-5).

3. Symptom Checklist 90 (SCL-90): is a checklist of diagnostic relevance symptoms.

4. 'Questionario per la valutazione delle organizzazioni di Significato Personale' (QSP): helps to find out which meaning organization belongs to a person. 
For each of these tests we collected both raw data and we calculated the summary indexes according to the tests guidelines. These indexes are shown to help the psychologists in order to have the 'Picture' of the patient at a glance.

\subsection{Data Sharing Platform}

This project originates from the idea of a group of psychologists who would like to improve their daily therapeutic routine with an IT-supported data sharing, since web platforms are the easiest and most effective tools for data sharing in the scientific and medical field $[1,2,3,4]$. An interactive web site was developed to give telematics support to type shared patient evaluations.

It is composed of two main parts: a SQL Server Database(DB), that stores anamnestic information and patient test results, and a website, which guarantees an easy and re- mote interaction with the DB. The access to patient's data is restricted by a login page and it is reserved to the psychologists involved in the project. The architecture is similar to other solutions developed in the laboratory of some of the authors, that are also routinely used in clinical practice $[5,6]$.

\subsection{Characteristics of the Sample}

In the period May 2017 - May 2021, a group of 248 patients were involved (130 women and $118 \mathrm{men}$ ) and the total number of compiled tests are 902. No discrimination was made regarding gender or occupation and the mean sample age was 41 years (range 3052). Questionnaires were first completed by patients on paper and then imported into the web platform by psychologists thanks to the support of pre-arranged spreadsheets.

\subsection{System of Anonymization}

The data collected for this research project were completely anonymous.

The patient is uniquely identified within the platform through a progressive number. The only anagraphic information stored about the patient are the birth sex and the year of birth. Each clinician maintains a matching between the unique identifier of the patient given by the platform and his anagraphic information. It's responsibility of the single physician to guarantee that no duplicates are inserted in the platform. Given the close cooperation with the doctors that enter the data, the authors are sure that this task is done correctly. Since the data is completely anonymous and unidentifiable, the GDPR does not apply.

\subsection{Missing Data Management}

As psychological tests were administered on paper and not all the answers were expressively mandatory, the collected dataset contained some missing values. This problem could be addressed in two ways: listwise deletion (or complete case analysis) by removing all the rows with a missing value from the dataset, or imputation methods by estimating the value of missing data. In order to keep the sample as big as possible, the second option was selected. 
According to literature, a generally effective algorithm in this scenario of data imputation is based on K-Nearest Neighbor (KNN). It imputes each sample's missing values using the mean value from $n$ nearest neighbors found in the dataset [7].

\subsection{Data Normalization}

Considering that the numerical values associated to each answer belonged to different scales, the second mandatory step in data pre-processing was normalization. In particular the chosen algorithm for data normalization was based on the Z-score:

$$
Z=\underset{\sigma}{x-\mu}
$$

where $\mu$ is the mean and $\sigma$ is the standard deviation.

The Z-score gives an idea of how far from the mean a data point is. It's a perfect strategy to deal with outliers because it measures how many standard deviations are below or above the population mean and raw score.

\section{Results}

\subsection{Web Tool}

After an authentication phase, the user can:

1. Decide if he/she wants to visualize data of a specific patient who has already been enrolled into the platform or add a new patient.

2. Store the scores of psychological tests through pre-formatted spreadsheets where the user has already manually inserted the numerical values corresponding to the patient's answers to the test.

3. Visualize a complete 'picture' containing all the relevant information about the patient and a summary of the scores obtained in each test ((Figure 1).

\subsection{Correlation between scales}

The developed system allows the automatic computation of the Pearson correlation coefficient between the indexes of the various scales. We considered 4 tests with a total number of 59 scales. In order to investigate the relations among this scales we calculated a complete correlation matrix. After a screening of this correlation matrix (1711 relations), we counted each correlation within the intervals defined by Akoglu in [8]. The obtained counts are summarized in Table 1. 


\begin{tabular}{|l|c|c|c|}
\hline \multicolumn{1}{|c}{ Tratti di Personalità } & Num_off_Quest & Totale & Average \\
\hline Anedonia & 8 & 8 & 1 \\
\hline Ansia & 9 & 18 & 2 \\
\hline Ricerca di Attenzione & 8 & 14 & 1,75 \\
\hline Insensibilità & 14 & 1 & 0,07 \\
\hline Inganno & 10 & 15 & 1,5 \\
\hline Depressivitá & 14 & 7 & 0,5 \\
\hline Distraibilità & 9 & 3 & 0,33 \\
\hline Eccentricità & 13 & 1 & 0,08 \\
\hline Labilità Emotiva & 7 & 9 & 1,29 \\
\hline Grandiosità & 6 & 6 & 1 \\
\hline Ostilità & 10 & 8 & 0,8 \\
\hline Impulsività & 6 & 0 & 0 \\
\hline Evitamento dell'Intimità & 6 & 1 & 0,17 \\
\hline Irresponsabilità & 7 & 3 & 0,43 \\
\hline Manipolatorietà & 5 & 9 & 1,8 \\
\hline Disregolazione Percettiva & 12 & 3 & 0,25 \\
\hline Perseverazione & 9 & 3 & 0,33 \\
\hline Affettività Ridotta & 7 & 8 & 1,14 \\
\hline Perfezionismo Rigido & 10 & 12 & 1,2 \\
\hline Tendenza a correre Rischi & 14 & 8 & 0,57 \\
\hline Angoscia di Separazione & 7 & 6 & 0,86 \\
\hline Sottomissione & 4 & 2 & 0,5 \\
\hline Sospettosità & 7 & 6 & 0,86 \\
\hline Convinzioni ed Esperienze & 8 & 11 & 1,38 \\
\hline Inusuali & 10 & 7 & 0,7 \\
\hline Ritiro & & & \\
\hline & & & \\
\hline
\end{tabular}

(a) PID-5 results table
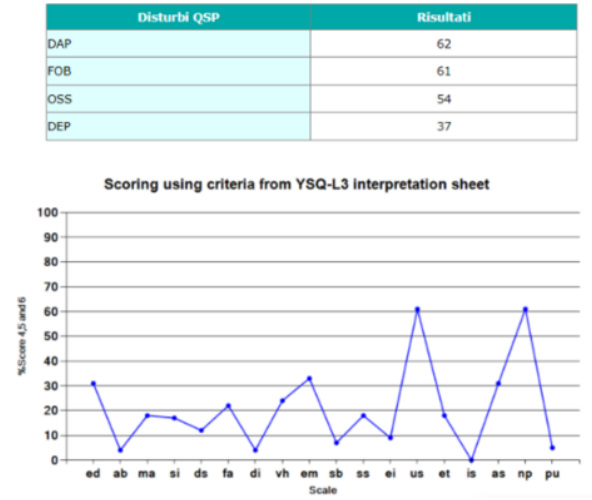

(b) The upper table refers to the personality traits extracted from the QSP questionnaire while the graph refers to the results of YSQL3 questionnaire.

Figure 1. Example of the personalized 'Picture' available for each patient and obtained collecting the summary results from the considered tests.

Table 1. Counts from correlation matrix.

\begin{tabular}{|c|c|c|}
\hline Correlation & Interval & Count \\
\hline Very strong positive correlation & 0.90 to 1.00 & 0 \\
\hline Strong positive correlation & 0.70 to 0.89 & 20 \\
\hline Moderate positive correlation & 0.40 to 0.69 & 371 \\
\hline Weak positive correlation & 0.20 to 0.39 & 760 \\
\hline Very weak positive correlation & 0.00 to 0.19 & 409 \\
\hline Very weak negative correlation & -0.19 to 0.00 & 61 \\
\hline Weak negative correlation & -0.39 to -0.20 & 5 \\
\hline
\end{tabular}

The minimum negative correlation found was -0.35 .

\section{Discussion}

During these years of application with the working group of some of the authors, they found that the reading of the exceptions is very useful. Specifically, an exception can be defined as the presence of unexpected correlations or moreover, as the absence of expected correlations (that are present in other patients in the sample with the same characteristics). For example, a patient who has a borderline mode of operation and who, unlike the norm, does not have traits of impulsiveness or emotional lability will immediately attract the attention of the clinician.

This kind of information can be achieved in a simple and quick way looking at the 'picture' that fully characterizes the individual patient. This attention translates into an attitude of research together with the patient of his specific functioning, with an 
attitude of respectful and real curiosity towards their personal way of being in addition to the diagnosis. These tools therefore allow the overcoming of the factors that unite the various patients, which becomes only a starting point for the therapeutic exploration of the specific characteristics of the patient. This in-depth work, in our opinion, is very valuable and innovative and would be impossible without the aid of the IT tool which, in addition to the immediate calculation of the correlations and their statistical significance, translates the correlation of the two variables taken into consideration with a graph giving the clinician an immediate vision of the patient with respect to others. This also makes it possible to detect similarities with other patients in the sample and therefore their comparison and to search for similarities and differences in the profile and style of functioning or in the anamnesis and life history. Examples of the previous are patients who have suffered a trauma or a precocious abandonment, significant emotional separations, significant medical pathologies, etc. Lastly, it should be noted that all patients in the sample were followed by therapists and they were clinical cases of which the therapist had deep knowledge and documentation.

\section{Conclusions}

It is our belief that the exploration of the potential of the IT tool as a clinical aid is only at the beginning. The graphic simplicity of consultation, the reliability of the tool and the ease and immediacy of consultation are its winning features. The calculated correlation indexes are under accurate study by a group of relevant psychologists (some of them are authors of this work), in order to define synergies and competitions between the used tests to improve their efficacy in the personalized diagnosis and treatment. The complexity and the amount of data that can be derived from it are the most promising characteristics for future research.

\section{References}

[1] Giacomini M, et al. Data modeling for tools and technologies for the analysis and synthesis of NANOstructures (TASNANO) project., Journal of Information Technology Research (JITR) 2.3 (2009): 49-70.

[2] Henrie A, et al. ClinVar Miner: Demonstrating utility of a Web-based tool for viewing and filtering ClinVar data., Human mutation 2018; 39(8): 1051-1060.

[3] Dyrlund TF, et al. MS Data Miner: A web-based software tool to analyze, compare, and share mass spectrometry protein identifications., Proteomics 2012; 12(18): 2792-2796.

[4] Hamosh A, et al. P heno DB: A New Web-Based Tool for the Collection, Storage, and Analysis of Phenotypic Features., Human mutation 2013; 34(4): 566-571.

[5] Giannini B, et al. From Liguria HIV Web to Liguria infectious diseases network: how a digital platform improved doctors' work and patients' care., AIDS research and human retroviruses 2018; 34(3): 239-240.

[6] Bonetto M, et al. I-Maculaweb: a tool to support data reuse in ophthalmology., IEEE journal of translational engineering in health and medicine 2015; 4: 1-10.

[7] Dong X, et al. TOBMI: trans-omics block missing data imputation using a k-nearest neighbor weighted approach. Bioinformatics 2019; 35(8): 1278-1283.

[8] Akoglu H. User's guide to correlation coefficients. Turk J Emerg Med. 2018;18(3):91-93. Published 2018 Aug 7. 